\title{
On the importance of including devolatilized stellar abundances in determining the composition of rocky exoplanets
}

Other Conference Item

Author(s):

Seidler, Fabian; Wang, Haiyang (1); Quanz, Sascha Patrick (D)

Publication date:

2021-04

Permanent link:

https://doi.org/10.3929/ethz-b-000523611

Rights / license:

Creative Commons Attribution 4.0 International

Originally published in:

EGUsphere, https://doi.org/10.5194/egusphere-egu21-15884 
EGU21-15884

https://doi.org/10.5194/egusphere-egu21-15884

EGU General Assembly 2021

(c) Author(s) 2022. This work is distributed under

the Creative Commons Attribution 4.0 License.

\title{
On the importance of including devolatilized stellar abundances in determining the composition of rocky exoplanets
}

\author{
Fabian Seidler, Haiyang Wang, and Sascha Quanz \\ ETH Zürich, Institute for Particle Physics and Astrophysics, Switzerland (fseidler@ethz.ch)
}

Since stars and their planets form from the same molecular clouds, stellar chemical composition can be informative, to first order, of planetary bulk chemistry. An important feature of terrestrial planets compared to their host stars is the depletion of volatiles, the most important being oxygen. Previous studies on planet interiors focus on the mass and radius constraints and/or the host stellar refractory elements (e.g. Fe, Si and $\mathrm{Mg}$ ), neglecting devolatilisation and its impact on the final picture of planet mineralogy and structure. This work assesses to what extent the devolatilised stellar abundances reflect rocky planetary composition.

We firstly test how the uncertainties associated with planetary mass and radius would affect the modelling results of core mass fraction - an important interior parameter. To do so, we choose the Sun-like star Kepler-21 (stellar abundance uncertainties $<0.05$ dex) as a case study and assume it hosts an Earth-mass-and-radius planet in its habitable zone. We then assign different levels of uncertainties to the mass and radius of the hypothetical planet, ranging from $0.1 \%$ to $20 \%$. We find that with increasing uncertainty level, the modelling result of core mass fraction constrained by the devolatilised stellar abundances and mass and radius becomes identical with the core mass fraction constrained purely by the devolatilised stellar abundances. This reveals the increased modelling degeneracy with growing uncertainties in mass and radius measurements, but also the strong constraints placed by using the devolatilised stellar abundances.

We further investigate a sample of 12 confirmed exoplanets, which are all less than 10 Earth masses and 2 Earth radii - i.e. potentially terrestrial planets or super-Earths - and with the measured uncertainties in mass and radius respectively less than $35 \%$ and $10 \%$. By comparing the prior and posterior distributions of mass and radius before and after introducing the devolatilised stellar abundances as another prior, we find that the posterior distributions of all samples, but 55 Cnc e and Kepler-107 c, can be sampled within the $2 \sigma$ ranges of the prior distributions. For the two exceptional cases, it means that the devolatilised stellar abundances and the measured mass and radius are not compatible within the level of $2 \sigma$.

We also find a diverse distribution of the core mass fractions of the sample from $0 \%$ (i.e. coreless) up to $40 \%$, which are consistent at the $2 \sigma$ level wwith the core mass fractions purely constrained by mass and radius measurements (except Kepler-107 c and $55 \mathrm{Cnc}$ e), but are significantly constrained by adding the devolatilized stellar abundances. In contrast, the previous study for the similar sample shows nearly constant core mass fractions of $\sim 30 \%$ based on the unaltered stellar 
abundances and by assuming 100\% Fe sunk into the core (i.e. free of consideration of the oxidation state of the planets). We emphasise that to break the degeneracies of terrestrial-type exoplanet interior modelling, we must use well the currently available observables including planetary mass and radius and host stellar chemical compositions, but they must be viewed through the lens of planet formation and the resulting devolatilization. 